\title{
Systematic Longitudinal Evaluating on boosting preschool children's food literacy
}

\author{
Guo $\operatorname{Tian}^{1}$, Liu weiwei ${ }^{2, *}$ \\ ${ }^{1}$ Chongqing Institute of Health Education, Yubei District, Chongqing \\ ${ }^{2}$ Medical and Social Development Research Center, School of Public Health and Management, Chongqing Medical University; National \\ Health and Social Risk Early Warning Collaborative Innovation Center Chongqing 400016
}

\begin{abstract}
To evaluate different methods in lifting food literacy which are applied to preschool children. Most articles are based on cross-sectional studies, an observational longitudinal design is seldom used, making it difficult to identify the true effectiveness of the methods. Thus, this article use a longitudinal method, including several online database. A systematic article based on longitudinal researches, by searching data in four electronic databases, Web of Science, PubMed, Elsevier (Science Direct) and Google Scholar, setting eligibility criteria, data selection, extracting data, data synthesis and quality assessment. Study identifies 771 original data, and after the de-duplication and eligibility selection, 9 studies are included in the article. Despite the small amount of literature applied, evidences are quiet useful. In comparison of caregiver-based intervention and classroom-based intervention, the later shows more effectiveness in short-term awareness training. Also, great potential has been found in technology-based intervention. The authors recommend future studies to focus on longitudinal research, so as to assess the potential of early childhood food literacy intervention, the mechanism and methods of these studies, the duration and effectiveness in different followup time etc.
\end{abstract}

\section{Introduction}

Diet-related diseases have been one of the most serious problems everlasting. It could arouse malnutrition, which results to a various of chronic diseases, such as gastroenteritis, cardiovascular diseases and overweight or obesity. Meanwhile, there is an increasing focus of dietrelated diseases. There has been numerous studies interpreted the relationship between diet-related behaviors and food literacy. Food literacy presents positive to support health. However, food literacy in many countries is insufficient. Studies prove that most eating behaviors are long-lasting, and are originated from the childhood experiences, both from the environment exposure and early cognitive characteristics.

Existing projects on early intervention to preschool children are based on the environment children exposed to. Most of them are based on a caregiver intervention and the school-based intervention. Both of them have proved to be effective, but most article and researches are based on cross-sectional cohort, the value of early intervention on food literacy can not be concluded accurately. Another novel category is to take advantage of the technology. But due to the lack of research quantum, it is only on the preliminary stage like investigating whether this kind of new strategy could be useful. Broadly speaking, these kind of researches are mainly applied to elder children and adolescents, yet it still has large upside potential to preschool children. The access to technology can be divided into more aspects than the other two interventions, and its assess to the the data can enlarge the subject size.

\section{Material and Methods}

Based on the concept of food literacy, this paper uses a systematic article methodology, through searching data, setting eligibility criteria, data selection, extracting data, data synthesis and quality assessment.

\subsection{Data sources and searched}

The following databases are sourced from four electronic databases, Web of Science, PubMed, Elsevier (Science Direct) and Google Scholar. Searches were restricted to human subjects, and English language, but there were no restrictions for publication data. For the purpose of the present article, 'preschool children' referred to individual aged from 3 to 6 years old, before entering primary schools, and 'food literacy' referred to 'a collection of inter-related knowledge, skills and behaviors required to plan, manage, select, prepare and eat foods to meet needs and determine food intake'. the outcome of food literacy is analyzed by food. Due to the concept of food literacy, the data base was searched using the key words or MeSH words: 'preschool children' in combination with 'school-based/care-center project',or 'parental/caregiver intervention',or

* Corresponding author: 1ww102551@cqmu.edu.cn 
'technology intervention', 'eating habits//fruit and vegetable intakes',or 'nutrition knowledge'

\subsection{Study Selection and Eligibility Criteria}

\section{Phase 1-Title and Abstract}

Age of children at baseline $\geqq 4$ and $\leqq 6$ years

Location of the study in high- or middle-income country (according to World Bank Standard...) Study design is observational longitudinal design Outcomes include adiposity/body mass, nutrition knowledge, food and vegetable intake and diet.

Phase 2 - Full Text

1.Age of children at baseline $(>3$, at most $<6)$

2.Location of the study in high- or middle-income country(according to World Bank Standard...)

3.Study design observational longitudinal design 4. There is between-children variation in the exposure 5.Outcomes include adiposity/body mass, nutrition knowledge, food and vegetable intake and diet.

Fig 1. Eligibility criteria

After removing the duplicates, records were screened in two phases using procedure (Figure 1). In phase one, title and abstracts were screened through the phase one eligibility criteria described in Figure 1. The full texts of all studies were screened through the phase two.In phase two, title and abstracts were screened through the phase two eligibility criteria described in Figure 1.

\section{Analysis of the general characteristics of research literature}

\subsection{Distribution of publication sources}

The number of papers included and excluded at each stage of the article process is reflected in Figure 2. The diagram shows overall number of references screened. Consequently, the final number of included studies are presented for behaviour outcomes only that were the focus of the current article_diet, adioposity/body mass, nutrition knowledge, food and vegetable intake.

Studies were included where participants were children aged $>3$ years and $<6$ years, and living in middleand high-income countries as defined by the World Bank ${ }^{[7]}$. Only observational longevity study designs, including case-control, prospective, and retrospective designs, were included. The exposure of interest was caregivers, caregiving centers, kindergartens, and technology, there was between-child variation in exposure, for example by timing of attendance (i.e., age when projects started.versus non-attendance. Studies were included where outcomes were objectively assessed or proxy/self-reported measures of diet, body mass index, nutrition knowledge, or fruit and vegetable intake. Studies excluded systematic articles.
After identified 771 articles, 494 deduplication articles were removed, 277 articles underwent title/abstract screening, and 47 full texts articles were articleed. 9 food literacy attributes met the eligibility criteria of the article and were included. Among these, two studies ${ }^{[6,15]}$ reported on adiposity/body mass, one study (6) reported on nutrition knowledge, four studies ${ }^{[1,3,4,14]}$ reported on fruit and vegetable intakes, six $(1,2,5,9,14,15)$ studies reported on diet. Some studies reported on more than one of the adiposity/body mass, nutrition knowledge, fruit and vegetable intakes, and diet.

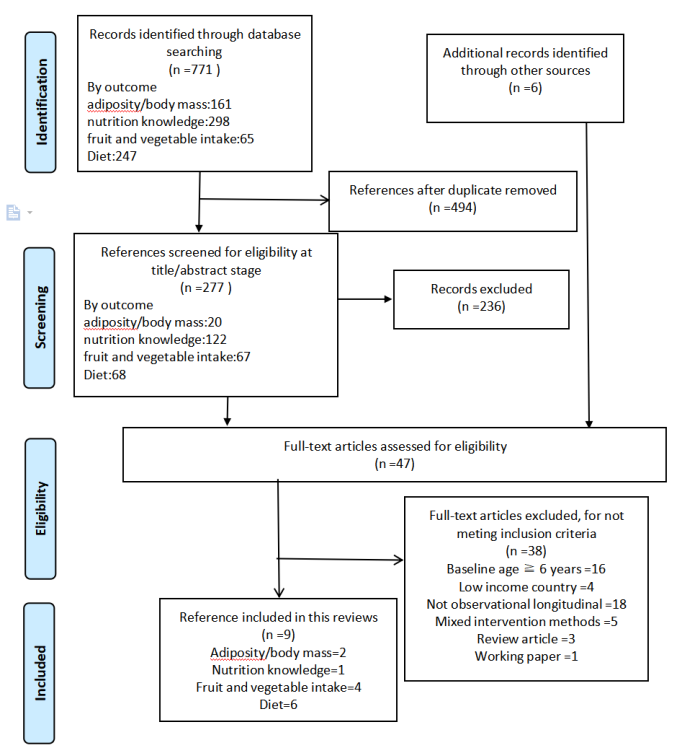

Fig 2. PRISMA diagram

\section{Summary of Included Studies}

A detailed description of each study's characteristics can be seen in Table 1. All included studies were from highincome countries, with five from the United States ${ }^{[1-4,9]}$, one from Portugal [5], one from South Korea ${ }^{[6]}$, one from the Netherlands ${ }^{[14]}$, and one from Japan ${ }^{[15]}$. Samples were generally related to children's gender (although one study did not mentioned) ${ }^{[4]}$, but varied greatly both in size (between 93 and 4232 subjects) and ethnic composition (between $0 \%$ and $100 \%$ Asian, with 2 studies did not mentioned the race/ethnicity or country of birth ${ }^{[4,5]}$, and one study providing information only for country of birth ${ }^{[14]}$. Included studies assessed exposure to caregivers, classrooms, or technology. Two studies were exposed to the intervention of caregivers ${ }^{[1,15]}$, three studies were exposed to classrooms' intervention ${ }^{[2,3,6]}$, four studies were exposed to technology ${ }^{[4,5,9,10]}$.

\subsection{Caregiver-based intervention effect}

Two studies evaluated the longitudinal relationship between childcare during early childhood. The conclusion are accordant, but the measurement and outcome perform differently. In Mizuki Sata et al. ${ }^{[15]}$ long term follow-up report, different caregiver present different impact on meal eating behavior and BMI, in the final report in 22 years, the subjects being cared for by grandparents at age 3 was associated with high prevalence of between-meal eating 
before dinner for boys and girls at ages 6 and 12 years, compared to children cared for by mothers. At age 22 years, boys cared for by grandparents had a higher prevalence of overweight than those cared for by mothers ( $18.5 \%$ versus $11.2 \%, \mathrm{P}=0.037$ ), but no such difference was noted in girls. However, both boys and girls cared for by grandparents had higher mean BMI over time than those cared for by mothers (coefficient $=0.47 \mathrm{~kg} / \mathrm{m} 2$ for boys and coefficient $=0.35 \mathrm{~kg} / \mathrm{m} 2$ for girls). In P. A. Williams et al. ${ }^{[1]}$ study, it took diet change as a key result. This paper found it had a substantial impact on children's at-home daily consumption of vegetables and use of lowfat/fat-free milk, and also had a significant increase in the frequency of child-initiated vegetable snacking after nutrition education for parents.

\subsection{Classroom-based intervention effect}

Three studies evaluated the longitudinal relationship between education intervention during early childhood. Two of them were both taken place in Head Start classrooms, one from the Mission X program. In different outcome variations, the key findings are similar, but the conclusion have different aspects. In Jieun Kim et al. ${ }^{[6]}$, study found that the mean differences in various anthropometric measures did not differ significantly between the groups adjusted for age, sex, and type of school. The Nutrition Quotient grades were significantly higher in the intervention group than the control group after the intervention $(\mathrm{p}=0.000)$. In summary, the 10week South Korean MX program improved the eating behaviors and nutrition status of young children. In $\mathrm{E}$ Smith et al. [3] report, they used carotenoid as a measurement, all groups demonstrated an increase in carotenoid scores, including the comparison group. But children who were assigned to treatment B (access fruit and vegetable consumption plus education) had significantly higher carotenoid values than those assigned to treatment A (access only fruit and vegetable consumption) and comparison group. Hence, it gave a clear picture of the effect of food education that such education perform better than passive feeding method. In Susan L. Johnson et al. ${ }^{[2]}$, both intervention and control groups demonstrated an increase in liking for the target food $(p=0.0001)$. However, the pattern of consumption of the target food was different, over time, for intervention and control groups $(\mathrm{p}<0.005)$. Children in the intervention group who liked the target food consumed nearly double their baseline consumption at postintervention $(\mathrm{p}<0.0001 ;)$ and maintained this increase at Y2 follow up $(\mathrm{p}<0.0001)$.

\subsection{Technology Intervention effect}

Four studies evaluated the longitudinal relationship between technology intervention during early childhood. Three of them ${ }^{[5,9,14]}$ use TV intervention, while one ${ }^{[4]}$ use the game intervention. In TV interventions, one ${ }^{[9]}$ use exposure of TV advertisement to measure the increase in negative eating and target brand eating, one ${ }^{[14]}$ use the influence of TV idol, and one ${ }^{[5]}$ in cartoon intervention.
Jennifer A. Emond et al. ${ }^{[9]}$ found that TV components of unhealthy food can increase the food choice on unhealthy food, and Parental dietary habits are strongly predictive of children's food intake. In comparison in Gertrude G. Zeinstra et al. ${ }^{[14]}$ and Sónia Gonçalves et al. ${ }^{[5]}$, Gertrude G. Zeinstra et al. [14] found the follow-up group had significant increase in target food intake rather than the intervention first ended. While in Sónia Gonçalves et al. ${ }^{[5]}$ found that after the intervention, the food choice will increase, but the preference on food will never change, and they also found there were no significant link between different ages. In the game intervention, although Brooke A. Jones et al. ${ }^{[4]}$ scored high in the bias evaluation, it still elucidated the possibility of game in early childhood intervention, the fruit and vegetable intake increase after the gamification education.

\section{Conclusions}

As far as we know, this study is the first systematic article of longitudinal studies about preschoolers food literacy intervention projects. Despite the few studies meeting the eligibility criteria, the including studies cover all three categories of the preschoolers food literacy intervention projects, caregiver-based intervention, classroom-based intervention and the technology intervention. The related studies acknowledge the suggestion in the protocol that classroom-based intervention is the most widely-used intervention methods and the most useful one in contrast to parental intervention, but the parental behavior influence most to child eating habit. Also, we found that children perform better under the form of individual learning rather than passive change (like passive feeding etc.).

Simultaneously, technology intervention perform positively in every researches, and it has the most potential to develop. A number of technology products have came into the market, more elements were added to this education field. In Brooke A. Jones's study [4], nutritioneducational game was firstly attempted to test on early childhood, and most newly updated technology (like touchscreen, 3-D technique[17] are mainly used in the intervention among adult and adolescents. Therefore, we suggest more technology-based intervention should be applied to preschoolers' food education.

This article uses longitudinal statistic to provide a better indication of causality between exposure and outcomes than cross-sectional studies.

But due to the low number of studies, for each of the outcomes did not allow us to present a meta-analyses. Heterogeneity in the study designs, definition of exposure, and the methods and measurement tools used also made it difficult to do comparisons.

Unlike the previous food education on preschoolers only benefit educated and well-off people[16], we suggest to apply more technology intervention, cause it's at low cost, widespread, and with no significant relationship to wealth.

When formulating policies, constitutors should think of the long-term impacts, started from early childhood till 
grown-ups, so that the interventions can perform better through a compulsory education.

In accordance to the study, we suggest that the future researches should try more and larger follow-up intervention or observation groups to compare, on account of each different types of intervention, and the measurements can be wiser if considered more individual aspects in combination of pre-surveys and results.

Overall, food literacy among preschoolers is important and the intervention programs are diversiform. Through analyzing the longitudinal researches, this systematic article unfolds the accessibility of the existing projects, and help identify policy, rules, regulations in different countries to support the nutrition education.

\section{Acknowledgments}

Chongqing Education Science "13th five year plan" project (2020-yq-06); The novel coronavirus pneumonia prevention and control emergency research (Management) special project (2020YJ01), Medical University Of Chongqing School of public health and management

\section{Reference}

1. Pamela A. Williams, Sheryl C. Cates, Jonathan L. Blitstein, James Hersey, Vivian Gabor, Melanie Ball, Katherine Kosa, Hoke Wilson, Sara Olson, Anita Singh, Nutrition Education Program Improves Preschoolers' At-Home Diet: A Group Randomized Trial, Journal of the Academy of Nutrition and Dietetics, Volume 114, Issue 7, 2014, Pages 10011008 , ISSN 2212-2672, https://doi.org/10.1016/j.jand.2014.01.015

2. Johnson, S.L., Ryan, S.M., Kroehl, M. et al. A longitudinal intervention to improve young children's liking and consumption of new foods: findings from the Colorado LEAP study. Int J Behav Nutr Phys Act 16, 49 (2019). https://doi.org/10.1186/s12966-0190808-3

3. Elizabeth Smith, Toto Sutarso, Gail L. Kaye, Access With Education Improves Fruit and Vegetable Intake in Preschool Children, Journal of Nutrition Education and Behavior, Volume 52, Issue 2, 2020, Pages 145151, ISSN 1499-4046, https://doi.org/10.1016/j.jneb.2019.07.016

4. Jones BA, Madden GJ, Wengreen HJ. The FIT Game: preliminary evaluation of a gamification approach to increasing fruit and vegetable consumption in school. Prev Med. 2014;68:76-79. doi:10.1016/j.ypmed.2014.04.015

5. Sónia Gonçalves, Rita Ferreira, Eva M. Conceição, Cátia Silva, Paulo P.P. Machado, Emma Boyland, Ana Vaz, The Impact of Exposure to Cartoons Promoting Healthy Eating on Children's Food Preferences and Choices, Journal of Nutrition Education and Behavior, Volume 50, Issue 5, 2018, Pages 451-457, ISSN 1499-4046, https://doi.org/10.1016/j.jneb.2017.12.015.
6. Kim J, Kim G, Park J, Wang Y, Lim H. Effectiveness of Teacher-Led Nutritional Lessons in Altering Dietary Habits and Nutritional Status in Preschool Children: Adoption of a NASA Mission X-Based Program. Nutrients. 2019;11(7):1590. Published 2019 Jul 13. doi:10.3390/nu11071590

7. The World Bank. World Bank Country and Lending Groups_-World Bank Data Help Desk. https://datahelpdesk.worldbank.org/knowledgebase/a rticles/906519-world-bank-countryand-lendinggroups (accessed on 18 January 2017).

8. 8. Obbagy, J.E.; Lyon, J.M.G.; Spahn, J.M. USDA Nutrition Evidence Library Systematic articles: Using food and nutrition research to inform nutrition programs and policies. Digest 2014, 49, 1-7.

9. Emond JA, Longacre MR, Drake KM, et al. Influence of child-targeted fast food TV advertising exposure on fast food intake: A longitudinal study of preschoolage children. Appetite. 2019;140:134-141. doi:10.1016/j.appet.2019.05.012

10. Prof. Debra L. Scammori \& Carole L. Christopher (1981) Nutrition Education with Children via Television: A article, Journal of Advertising, 10:2, 26-36, DOI: 10.1080/00913367.1981.10672758

11. Morgan McCloskey et al., Parent Perceptions of Mobile Device Use Among Preschool-Aged Children in Rural Head Start Centers, Journal of Nutrition Education and Behavior, Volume 50, Issue 1, 2018, Pages 83-89.e1, ISSN 1499-4046, https://doi.org/10.1016/j.jneb.2017.03.006.

12. R Hedaoo, S Val.Nutrition education \& child health care a cognitive approach using multimedia technology. Advanced Studies in Biology, Vol. 7, 2015, no. 1, 39-48.

http://dx.doi.org/10.12988/asb.2015.41053

13. YIEN, Jui-Mei; Hung, Chun-Ming et al. A GameBased Learning Approach to Improving Students' Learning Achievements in a Nutrition Course. TOJET: The Turkish Online Journal of Educational Technology, April 2011, volume 10 Issue 2. ISSN1303-6521. https://eric.ed.gov/?id=EJ932220

14. Gertrude G. Zeinstra, Valesca Kooijman, Stefanie Kremer (2017). My idol eats carrots, so do I? The delayed effect of a classroom-based intervention on 4-6-year-old children's intake of a familiar vegetable, Food Quality and Preference, Volume 62, 2017, Pages 352-359, ISSN 0950-3293, https://doi.org/10.1016/j.foodqual.2016.11.007.

15. Mizuki Sata et al. (2015)Impact of Caregiver Type for 3-Year-Old Children on Subsequent Between-Meal Eating Habits and Being Overweight From Childhood to Adulthood: A 20-Year Follow-up of the Ibaraki Children's Cohort (IBACHIL) Study. J Epidemiol 2015;25(9):600-607. doi:10.2188/jea.JE20140078

16. Kimura, A.H. Food education as food literacy: privatized and gendered food knowledge in contemporary Japan. Agric Hum Values 28, 465-482 (2011). https://doi.org/10.1007/s10460-010-9286-6 
17. Johnston, Jeanne \& Massey, Anne \& DeVaneaux, Celeste. (2012). Innovation in Weight Loss Intervention Programs: An Examination of a 3D Virtual World Approach. Proceedings of the Annual Hawaii International Conference on System Sciences. 2890-2899. 10.1109/HICSS.2012.325. 